Www.jmscr.igmpublication.org

Index Copernicus Value: 79.54

ISSN (e)-2347-176x ISSN (p) 2455-0450

crossrefDOI: https://dx.doi.org/10.18535/jmscr/v7i2.72

Journal Of Medical Science And Clinical Research

IGM Publication

An Official Publication of IGM Publication

\title{
Diagnostic and Prognostic evaluation of meningioma by IHC markers- A study of 100 cases
}

\author{
Authors \\ Prof. Dr Shikha Ghanghoria, Dr Paresh Sodhiya, Dr Vijeta Niranjan \\ Dr Varsha Argal, Prof. Dr Arvind Ghanghoria* \\ *Corresponding Author
}

Prof. Dr Arvind Ghanghoria

M.G.M. Medical College Indore, MP, India

Email: profdrarvindghanghoria@gmail.com

\begin{abstract}
Introduction: Meningioma it is basically a tumour originating from meninges as said by by Cushing in 1921. It is typically a benign and slow growing tumour which appears mostly in the late decades of life.

Aim: 1) To evaluate histological subtypes of meningioma according to WHO classification. 2)To evaluate various immunohistochemical markers in meningioma. 3) To evaluate proliferative markers (Ki-67 LI) and correlate it with various histological grades of meningioma.

Materials and Methods: We have studied 100 cases of meningioma over a period of 5 years. Menigioma was diagnosed on the basis of CT Scan, MRI and by histopathological examination, we have correlated it with age and sex distribution, histological type and WHO grading of tumour.IHC markers EMA, vimentin, Pan CK and S100 were applied and confirmation of diagnosis was done, Further the prognosis was evaluated with the help of Ki67.

Results: As per the results meningiomas are more common in females (60\%), commonest age group of this tumor is found to be from forth to sixth decade of life. The commonest histopathological type was meningotheliomatous meningioma. The $96 \%$ of the meningioma was WHO GRADE I tumour consist of meningotheliomatous meningioma. EMA was expressed by $94 \%$ of all meningiomas, Vimentin positivity seen in all cases of meningioma suggestive of mesenchymal origin, Cytokeratin AE1/AE3 was expressed in $4 \%$ of meningiomas and involved predominantly secretory meningiomas(80\%). S-100 positivity was seen in $26 \%$ cases. $K i-67$ was positive in all the cases of anaplastic meningiomas.

Conclusion: Conclusion drawn show the mean age of meningioma is around 48 years with female preponderance, It does not corresponds with variants and grade of the tumor. Biological behavior of the tumor is studied through 2016 WHO grading system of CNS tumors. Along with the histomrophological features Immunohistochemistry markers i.e. EMA, VIMENTIN, \& S-100 should be considered as the diagnostic tool for meningiomas

Keywords: Menigioma, WHO grade, IHC, $K i-67 / M I B-1$ index.
\end{abstract}

\section{Introduction}

Meningiomas are primary CNS tumors that arise from the meningothelial cells. They constitute
$30 \%$ of primary intracranial tumours ${ }^{[1]}$. They are generally slow growing benign tumours with predilection for women ${ }^{[2]}$. 
World Health Organization (WHO) defines meningiomas as a group of mostly benign, slow growing neoplasms that are most likely derived from the meningothelial cells of the arachnoid layer $^{(3)}$.

According to the World Health Organization (WHO) criteria, meningiomas are classified into 3 grades $^{(4)}$. Grade I meningiomas are the most frequent and benign with a low risk of recurrence. Grade II meningiomas are less common with higher rate of recurrence, and grade III tumors are rare. Different WHO subtypes are there among them the most commonly encountered are the meningothelial, fibrous, and transitional meningiomas $^{(4)}$.

Although conventional methods are the mainstay for pathologic diagnosis, Currently, the most commonly used IHC markers for the diagnosis of meningiomas are epithelial membrane antigen (EMA) and progesterone receptor (PR), CD34 for the diagnosis of SFT/HPC, and S100 for the diagnosis of schwannoma. However, these markers have suboptimal sensitivities and specificities and vary with regard to the grades or subtype of meningioma ${ }^{(4,5-7)}$.

Proliferation markers include p53, Ki-67 (MIB-I), argyrophilic nucleolar organizer regions (AgNOR), 5-bromo-2'-deoxyuridine (BrdU) and proliferating cell nuclear-antigen (PCNA) [8]. Among these proliferation markers Ki67 labeling index is more valuable as it is expressed in all active phases of cell cycle (G1, S, G2 \& M) except Go phase ${ }^{[9]}$.

The present study was performed to correlate the histologic subtype and to investigate the expression of mesothelioma-related markers in meningiomas and to determine the usefulness of proliferative markers (Ki-67) in the grading of intracranial meningiomas, so as to objectively predict the biological behaviour.

\section{Materials and Methods}

A prospective and retrospective study was conducted in the histopathology section of department of pathology M.G.M. Medical College
Indore. All the patients with primary intracranial and intraspinal meningiomas which were operated in our institute and then diagnosed in the Department of Pathology during the period from June 2013- June 2018 were included in our study. The study was approved by the institutional ethics committee (IEC). The details of the each patient were taken from medical records i.e. age, gender, clinical presentation, radiological evaluation, location, brain infiltration and recurrence were noted. The histological sections were reviewed and all tumors were graded according to WHO 2016 criteria. For the evaluation of prospective cases, the received samples were firstly fixed in 10 percent formalin for 24 hours. If the resected tissue was received as fragmented bits, all the tissues were submitted for processing. If the tumor size exceeded $6-8 \mathrm{cms}$, representative sections were taken. After the routine processing of tissue the paraffin embedded blocks were cut into 4-5 micron sections and stained with routine Haematoxylin and Eosin stain (H\&E).

Following this the sub typing and grading was done according to WHO (2016) grading system.

Immuno histochemical (IHC) staining was performed on all prospective and recent retrospective cases. For immune histochemistry a $3 \mu \mathrm{m}$ thick, formalin fixed, paraffin embedded tissue sections were cut and mounted on precoated poly lysine slides.

Horse radish Peroxidase (HRP) Method was used in this study, which uses a non-biotin polymeric technology wherein the secondary antibody conjugated to Poly-HRP reagent is bound to the primary antibody and is visualized by the diaminobenzidine (DAB) chromogen. Antigen retrieval was done with the help of retrieval chamber. For retrospective cases, stored slides were taken out and reviewed for sub typing and grading. Wherever necessary blocks were cut and fresh slides prepared. One of the representative blocks were selected for IHC staining. The Primary antibodies which were included in our study are EMA (Epithelial Membrane Antigen), 
Vimentin, Pan CK, S-100. Ki 67 LI taken as proliferative marker.

\section{Evaluation of Immunohistochemical Staining}

The proportion score is calculated as :

Score 0 - no immunoreactive cells,

Score $1-1 \%-25 \%$,

Score $2-26 \%-50 \%$, and

Score 3 - more than $50 \%$ immunoreactive cells.

The staining intensity was graded as :

Score 1 - weak

Score 2 - moderate

Score 3 - intense
A total score was calculated by adding the two above mentioned scores i.e proportion score and intensity score.

In addition the staining pattern (whether cytoplasmic +/or membranous) was recorded.

For Ki-67 scoring all the cells with brown nuclear staining were considered as positive. The Ki-67 labelling index (Ki-67 LI) was considered as the percentage of positively stained tumour nuclei per 1000 tumour cells.

Statistical Analysis- The statistical analysis done in this study was proportion test for using Pearson chi-square test, SPSS version 21 (trial version) for $\mathrm{Ki}-67 \mathrm{LI}$. The p-value of $<0.05$ is considered to be statistically significant.

\begin{tabular}{|cl|c|c|}
\hline \multicolumn{3}{c}{ Correlations } \\
\hline \multirow{3}{*}{ Kiscore } & Ki score & Grade \\
& Pearson Correlation & 1 & $1.000^{* *}$ \\
& Sig. (2-tailed) & & .000 \\
& $\mathrm{~N}$ & 100 & 100 \\
& Pearson Correlation & $1.000^{* *}$ & 1 \\
Grade & Sig. (2-tailed) & .000 & \\
& $\mathrm{~N}$ & 100 & 100 \\
\hline
\end{tabular}

**. Correlation is significant at the 0.01 level (2-tailed).

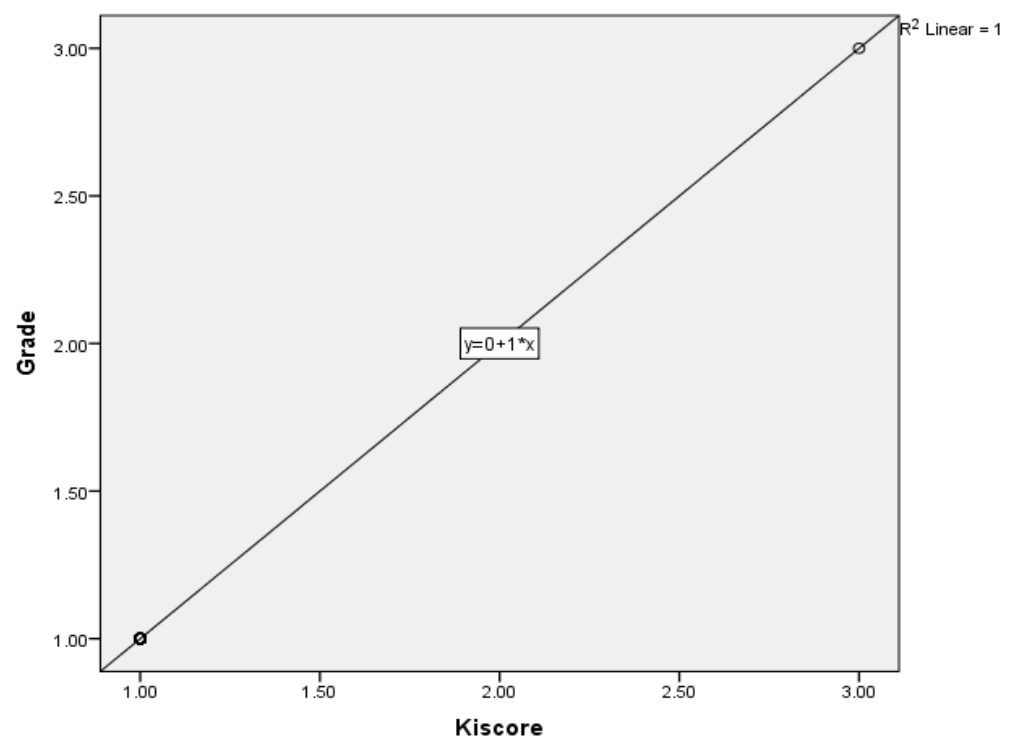

sex * type Cross tabulation

Count

\begin{tabular}{|ll|c|c|c|c|c|c|c|c|}
\hline & & \multicolumn{9}{|c|}{ type } & \multirow{2}{*}{ Total } \\
\cline { 3 - 9 } & & 1.00 & 2.00 & 3.00 & 4.00 & 5.00 & 6.00 & 7.00 & \\
\hline \multirow{2}{*}{ sex } & 1.00 & 32 & 15 & 7 & 6 & 5 & 2 & 1 & 68 \\
\multirow{2}{*}{ Total } & 2.00 & 18 & 6 & 3 & 3 & 2 & 0 & 0 & 32 \\
& & 50 & 21 & 10 & 9 & 7 & 2 & 1 & 100 \\
\hline
\end{tabular}


sex * Grade Cross tabulation

Count

\begin{tabular}{|ll|c|c|c|c|}
\hline & & \multicolumn{3}{|c|}{ Grade } & \multirow{2}{*}{ Total } \\
\cline { 3 - 5 } & & 1.00 & 2.00 & 3.00 & \\
\hline \multirow{2}{*}{ sex } & 1.00 & 66 & 1 & 1 & 68 \\
\multirow{2}{*}{ Total } & 2.00 & 30 & 2 & 0 & 32 \\
\hline
\end{tabular}

sex * Ki score Cross tabulation

Count

\begin{tabular}{|lc|c|c|c|c|}
\hline & & \multicolumn{3}{|c|}{ Kiscore } & \multirow{2}{*}{ Total } \\
\cline { 3 - 5 } & & 1.00 & 2.00 & 3.00 & \\
\hline \multirow{2}{*}{ sex } & 1.00 & 66 & 1 & 1 & 68 \\
\multirow{2}{*}{ Total } & 2.00 & 30 & 2 & 0 & 32 \\
\hline
\end{tabular}

\section{Results}

Table 1 - Showing age wise distribution of cases

\begin{tabular}{|l|c|}
\hline AGE GROUPS & NO. OF CASES \\
\hline $0-20$ & 0 \\
\hline $20-40$ & 3 \\
\hline $40-60$ & 96 \\
\hline $60-80$ & 1 \\
\hline$>80$ & 0 \\
\hline
\end{tabular}

Table 2 Showing sex wise distribution of cases

\begin{tabular}{|l|c|}
\hline SEX & PERCENTAGE OF CASES \\
\hline FEMALE & $62 \%$ \\
\hline MALE & $38 \%$ \\
\hline
\end{tabular}

Table 3 Showing grade wise distribution of cases

\begin{tabular}{|l|c|}
\hline GRADE & PERCENTAGE \\
\hline GRADE 1 & $96 \%$ \\
\hline GRADE 2 & $03 \%$ \\
\hline GRADE 3 & $01 \%$ \\
\hline
\end{tabular}

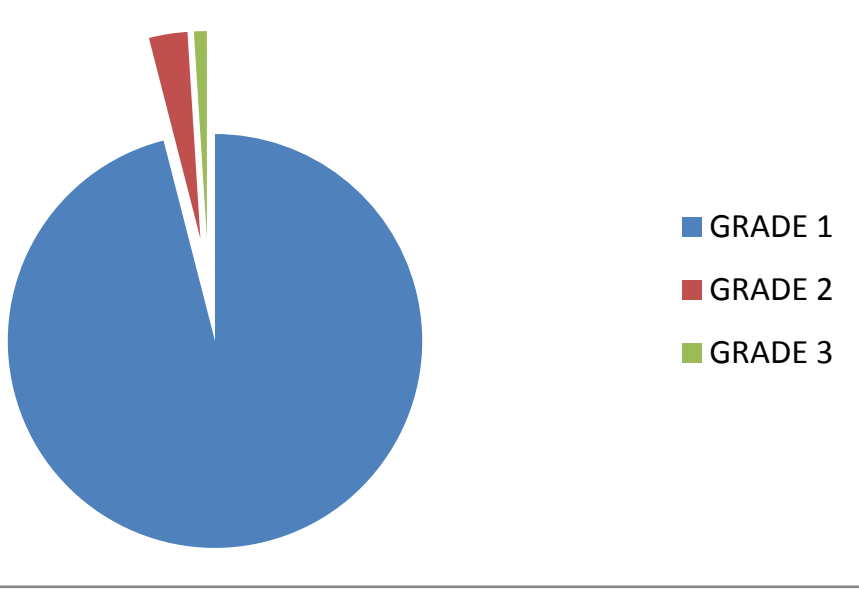




\section{JMSCR Vol||07||Issue||02||Page 382-390||February}

Table 4 Commonest variants of Meningioma

\begin{tabular}{|l|c|}
\hline Type Of Meningiomas & Number of Cases \\
\hline Meningothelial & 50 \\
\hline Psammomatous & 21 \\
\hline Transitional & 10 \\
\hline Angiomatous & 9 \\
\hline Fibroblastic & 7 \\
\hline Atypical & 2 \\
\hline Anaplastic & 1 \\
\hline
\end{tabular}

Grade I meningiomas were most common (96.0\%) followed by grade II (3.0\%) and grade III (1.0\%. Meningothelial (50.0\%) meningioma was the commonest subtype followed by psammomatous (21.0\%), transitional (10.0\%), angiomatous
(9.0\%), fibroblastic (7.0\%). Grade II meningiomas included atypical (3 cases) and Grade III meningioma include anaplastic (1 case) meningiomas.

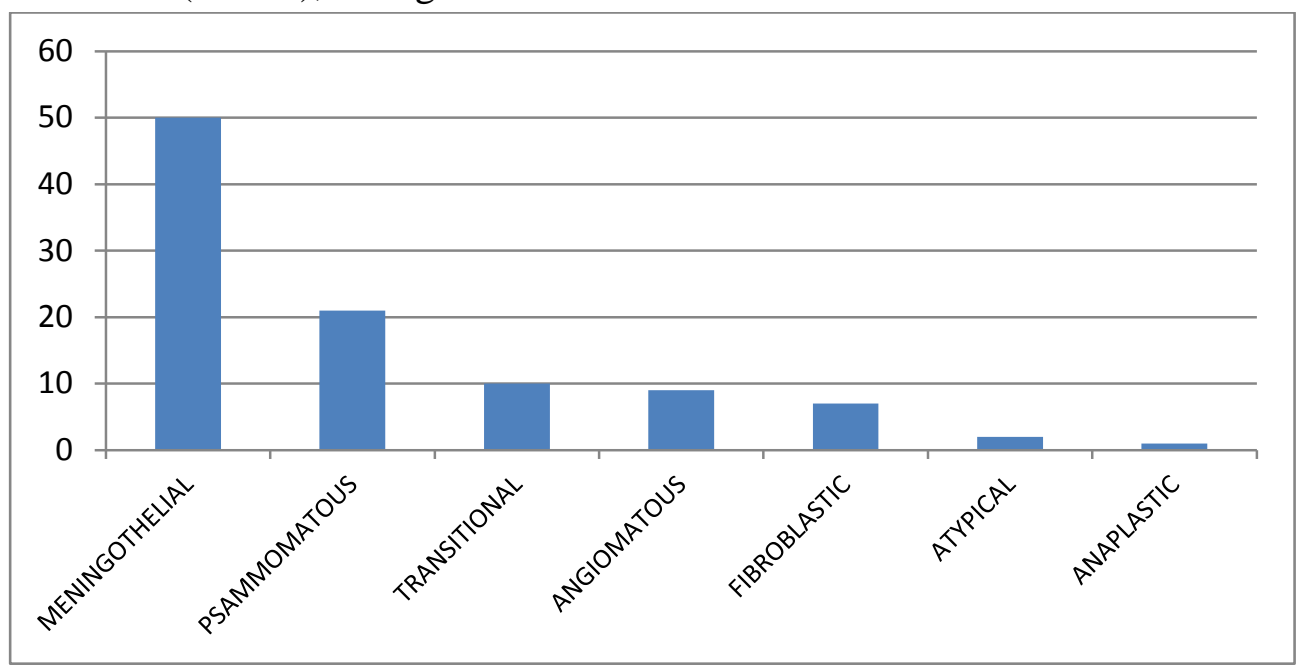

Fig. 1 Bar graph showing common variants of meningioma

Among the total 100 meningioma cases, which included 40 prospective cases and 60 retrospective cases, the majority of meningioma cases were fall under 40 to 60 year age group. There were 68 females and 32 males. In all cases of meningiomas including aggressive variants there was a female predominance $(\mathrm{F}: \mathrm{M}=2.1: 1)$.

All the studied cases were cranial in location. Neuroimaging and operative data showed that 92\% had supratentorial tumors and $8 \%$ had infratentorial tumors.

Photomicrographs

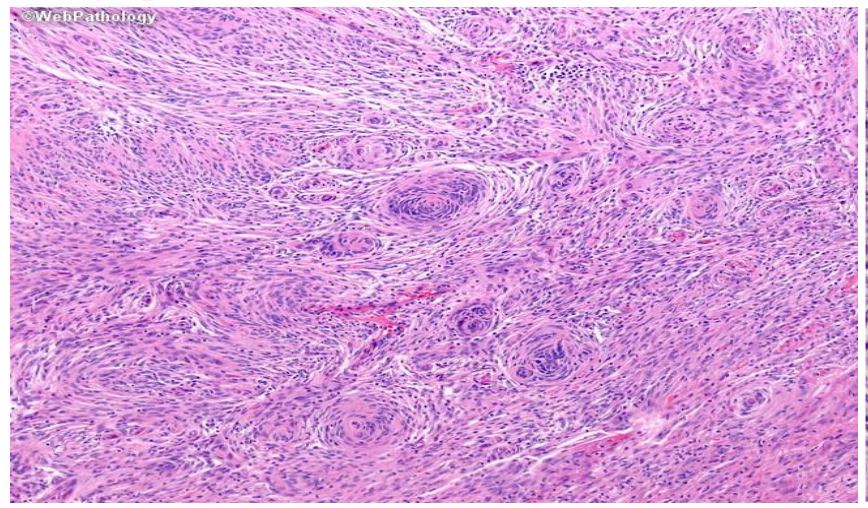

A

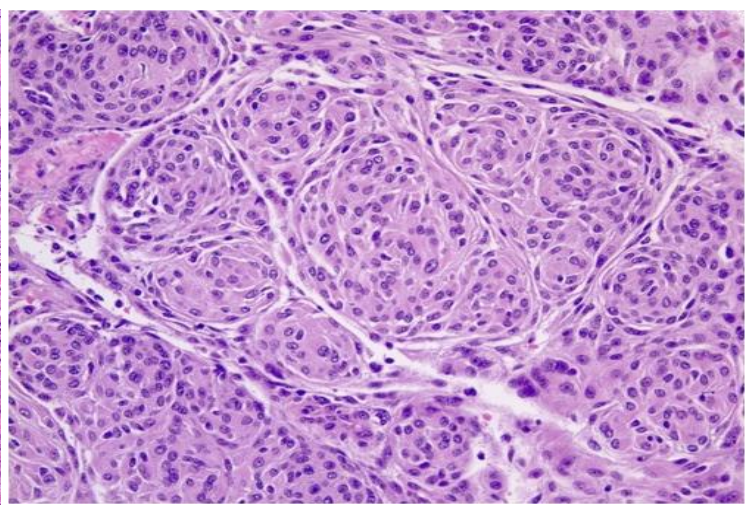

B

Fig 2. A -Showing low power view (10x) of meningioma B-showing high power view (40x) of meningotheliomatous meningioma 


\section{JMSCR Vol||07||Issue||02||Page 382-390||February}

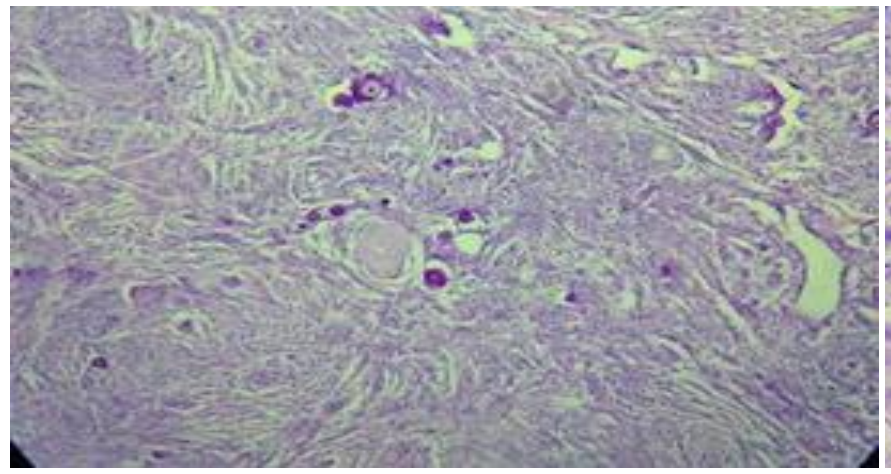

A

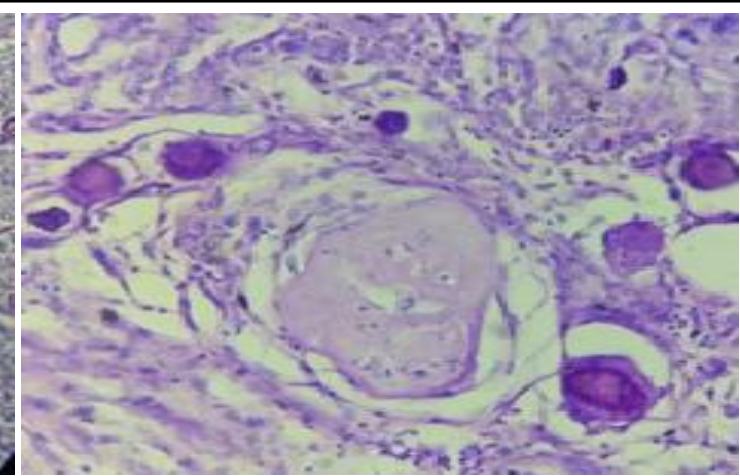

B

Fig 3 A -Showing low power view (10x) of meningioma B-showing high power view (40x) of transitional meningioma

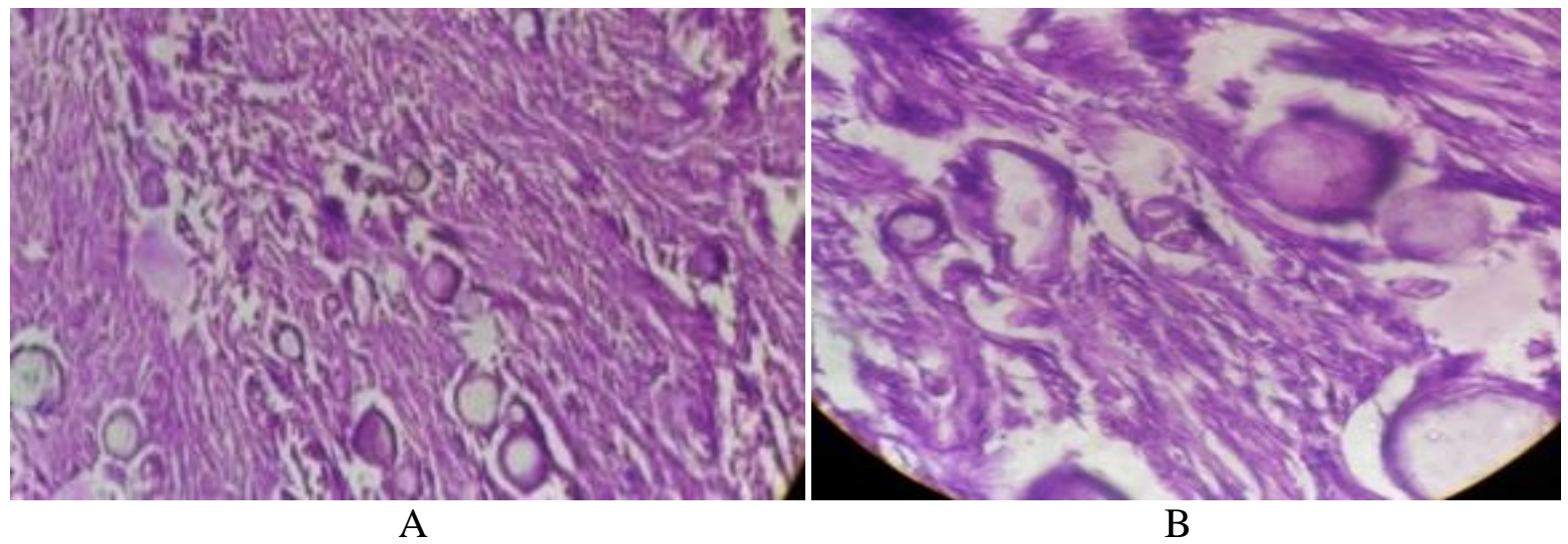

Fig 4 A Showing low power view (10x) of meningioma B-showing high power view (40x) of Psammomatous meningioma

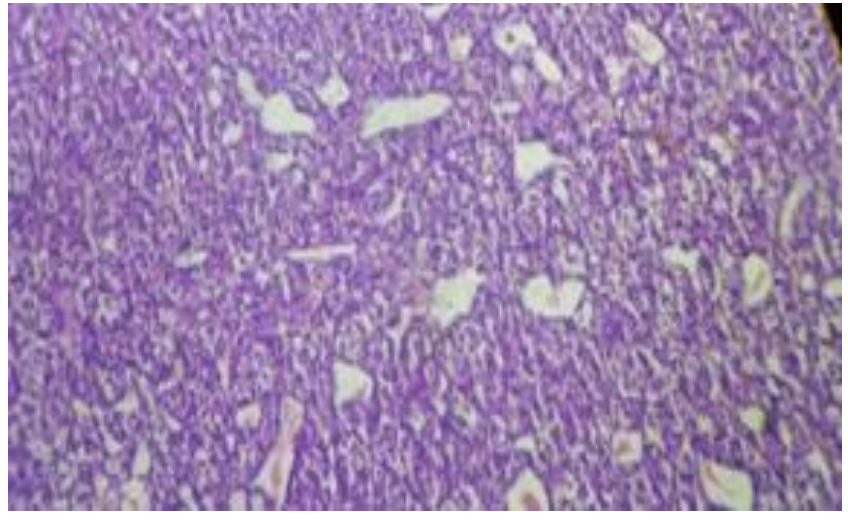

A

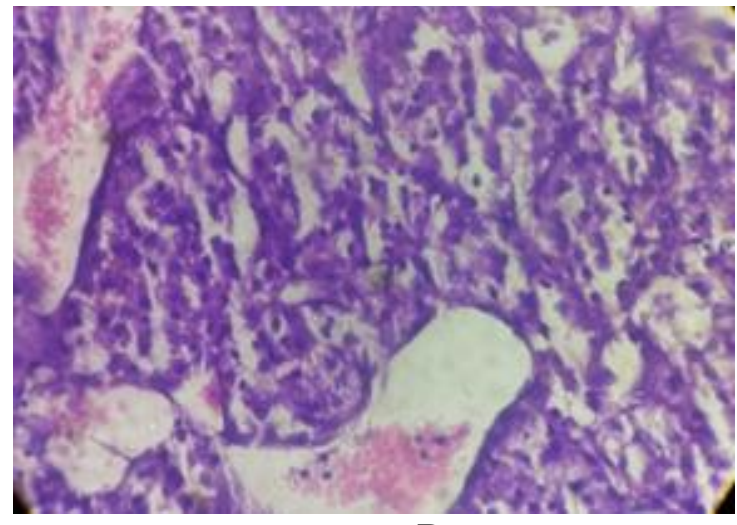

B

Fig 5 A Showing low power view (10x) of meningioma B-showing high power view (40x) of Angiomatous meningioma

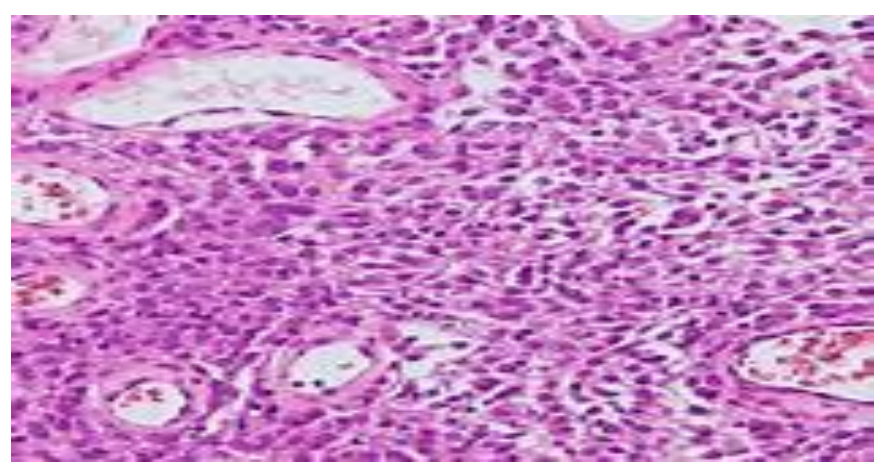

Fig 6 -Showing high power view(40x) of Atypical meningioma 


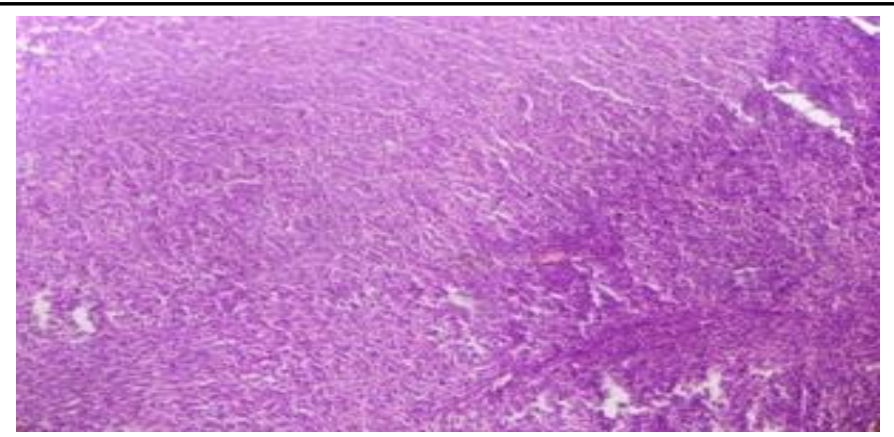

Fig 7 -Showing high power view (40x) of Anaplastic meningioma

\section{Immunohistochemistry Markers-}

Immunohistochemistry markers which were applied are EMA, PAN CYTOKERATIN, S-100, GFAP, VIMENTIN, NFP and KI 67. About 94\% cases are positive for EMA and Vimentin is positive in $87 \%$ cases while PAN CK (AE1/AE3) $4 \%$ cases. S-100 positvity seen in $43 \%$ cases while GFAP and NFP are found to be negative.
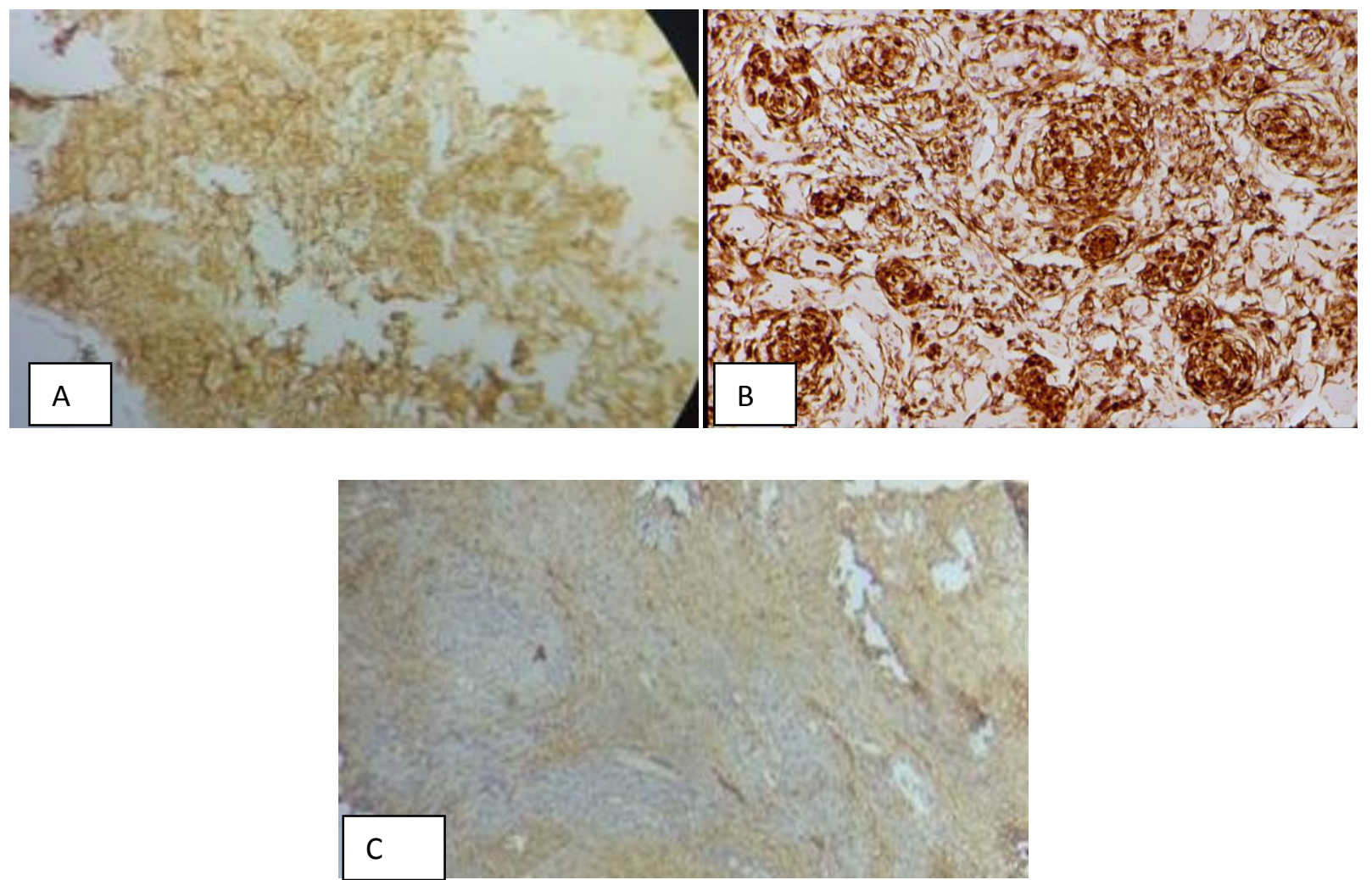

Fig 6- A -Showing EMA positivity, B- showing Vimentin positivity, C - showing S-100 positivity

\section{Ki-67 Labelling index (Ki-67 LI)}

Ki-67 scoring is more with grade III meningiomas followed by grade II meningiomas, while grade 1 meningiomas are showing only $1-2 \%$ of $\mathrm{Ki}$ positive cells. Meningiomas were distributed according to the grade as Most of the grade I have Ki-67 LI $\leq 7$ in contrast to grade II and grade III meningiomas which have LI $\geq 7$. Ki-67 LI for grade I, grade II and grade III meningiomas were $3.1 \%, 7 \%$ and $14.2 \%$ respectively.

\section{Discussion}

In our study, we concluded that, Grade I meningiomas were most common (96.0\%) followed by grade II $(3.0 \%)$ and grade III $(1.0 \%)$. Mean Ki-67 LI in grade I, grade II and grade III meningiomas were $3.1 \%, 7 \%$ \& $14.2 \%$ respectively. As per the results of the study correlations between histological variants of meningiomas and immunohistochemistry could not be found. EMA, VIMENTIN, and S-100 are 
positive in majority of the menigiomas while few cases show AE1/AE3 positivity. This is similarly explained by the conclusion drawn by Mennel et al. who stated that despite of multiple variants cellular form of meningiomas are similar.

Ramesh Babu Telugu et al in Histopathological and Immunohistochemical Evaluation of Meningiomas with Reference to Proliferative Markers p53 and Ki-67 found that the common age group for all grades of meningioma was 5 th 6th decade and declining and there were 78 males and 146 females. Almost similar results were found in our study with maximum cases fall under 40 to 60 year age and maximum cases were female. ${ }^{(10)}$

Ramesh Babu Telugu et al in Histopathological and Immunohistochemical Evaluation of Meningiomas with Reference to Proliferative Markers p53 and Ki-67 also found that Grade I meningiomas were most common $(86.6 \%)$ followed by grade II (10.7\%) and grade III (2.7\%) which is similar with our study. ${ }^{(10)}$

Recently Roser et al., reported a large retrospective study of 600 resected meningiomas in which histological grading revealed $91 \%$ WHO grade I meningioma (mean MIB-1 LI: 3.88\%), 7\% grade II meningioma (mean MIB-1 LI: 9.95\%) and $2 \%$ grade III meningioma (mean MIB-1 LI: $12.18 \%$ ) which is similar to our study. ${ }^{(11)}$

Camille Boulagnon-Rombi et al in Immunohistochemical Approach to the Differential Diagnosis of Meningiomas and Their Mimics found that AE1/AE3 was expressed in 6\% (7/116) of meningiomas, and involved predominantly secretory meningiomas $(80 \%, 4 / 5)$. Weak and focal positivity was observed in $5 \%$ of meningothelial and transitional meningiomas (both 1/19) and in $4 \%$ of atypical meningiomas (1/26). Camille also found that EMA was expressed by $90 \%(113 / 126)$ of all meningiomas there was no statistical difference between grades and types ( $\mathrm{p}^{1 / 40.75}$ and 0.23 , respectively, v2 test) where as in our study we have found that EMA was positive in $94 \%$ of cases. ${ }^{(12)}$
In our study we have found S-100 positvity in $43 \%$ while GFAP and NFP are found to be negative similar results were found in a study done by Camille Boulagnon-Rombi et al in Immunohistochemical Approach to the Differential Diagnosis of Meningiomas and Their Mimics i.e. around $34 \%$ cases shows positivity for $\mathrm{s}-100 .^{(12)}$

\section{Conclusion}

Conclusion drawn show the mean age of meningioma is around 48 years with female preponderance, It does not corresponds with variants and grade of the tumor. Biological behavior of the tumor is studied through 2016 WHO grading system of CNS tumors. Along with the histomrophological features Immunohistochemistry markers i.e. EMA, VIMENTIN, \& S-100 should be considered as the diagnostic tool for meningiomas.

As EMA is positive in almost all the cases of most of the studies, it can be selected as most specific diagnostic marker for meningiomas. Ki-67 labelling should be done for evaluation of proliferative activity and for consideration of high grade meningiomas which will be helpful in management and to find out the prognosis of menigiomas. Ki-67 LI is the most important criteria for distinguishing anaplastic meningioma from those of benign meningioma.

\section{References}

1. CBTRUS. Statistical Report: Primary brain tumours in the United States, 19982002. Publis. Tumour Registry of United States. 2005. Perry A, Louis DN, Scheithauer BW, Budka H, Deimling VA. Meningiomas.

2. In: Louis DN, Ohgaki H, Wiestler OD, Cavenee WK, editors. World Health Organization classification of the central nervous system. 2007:164-72.

3. Rao S, Sadiya N, Doraiswami S, Prathiba D. Characterization of morphologically benign biologically aggressive 
meningiomas. Neurol India. 2009;57:74448. [PubMed]

4. Sushila Jaiswal in Meningiomas: Objective assessment of proliferative indices by immunohistochemistry and automated counting method

5. Perry A, Louis DN, Budka $\mathrm{H}$, et al. Meningioma. In: Louis DN, Ohgaki H, Wiestler OD, Cavenee WK, eds. WHO classification of tumours of the central nervous system, Fourth Edition revised. Lyon, France: IARC press 2016:232-45

6. Mezmezian MB, Carassai MB, Dopazo V, et al. Immunohistochemical expression of progesterone receptors in nonmeningothelial central nervous system tumors. Appl Immunohistochem Mol Morphol 2016;

doi: 10.1097/PAI.0000000000000318

7. $\mathrm{Ng} \mathrm{J}$, Celebre A, Munoz DG, et al. Sox 10 is superior to $\mathrm{S} 100$ in the diagnosis of meningioma. Appl Immunohistochem Mol Morphol 2015;23: 215-9

8. Agaimy A, Buslei $\mathrm{R}$, Coras $\mathrm{R}$, et al. Comparative study of soft tissue perineurioma and meningioma using a five-marker immunohistochemical panel. Histopathology 2014;65:60-70

9. Langford LA, Cooksley CS, DeMonte F. Comparision of MIB-1 (Ki-67) antigen and bromodeoxyuridine proliferation indices in meningiomas. Hum Pathol. 1996;27:350-54. [PubMed]

10. Habberstad AH, Gulati S, Torp SH. Evaluation of the proliferation markers $\mathrm{Ki}$ 67/MIB-1, mitosin,survivin, pHH3, and DNA topoisomerase IIain human anaplastic astrocytomas - an immunohistochemical study. Diagn Pathol. 2011;6:43. [PMC free article] [PubMed]
11. Ramesh Babu Telugu et al in Histopathological and Immunohistochemical Evaluation of Meningiomas with Reference to Proliferative Markers p53 and $\mathrm{Ki}-67$

12. Roser F, Samii M, Ostertag H, Bellinzona M. The Ki-67 proliferation antigen in meningiomas. Experiencein 600 cases. Acta Neurochir (Wien). 2004;146:37-44.

13. Camille Boulagnon-Rombi et al in Immunohistochemical Approach to the Differential Diagnosis of Meningiomas and Their Mimics. 